\title{
Erratum: Size effects in long-term quasistatic heat transport [Phys. Rev. E 87, 062118 (2013)]
}

\author{
George Y. Panasyuk and Kirk L. Yerkes
}

(Received 14 April 2019; published 13 May 2019)

\section{DOI: 10.1103/PhysRevE.99.059902}

In Eq. (50), a factor " $1 / 2$ " on the left hand side was lost. Corrected Eq. (50) is

$$
\frac{1}{2} \frac{d}{d t} \sum_{k=1}^{N}\left\langle E_{1 k}\right\rangle=-\frac{\hbar \hat{\gamma} D^{2}}{2 \pi} \sum_{k=1}^{N} \frac{\Delta v_{k}^{2} G\left(v_{k}, t\right)}{v_{k}^{2}+D^{2}}\left(n_{1 k}-n_{2 k}\right),
$$

and after multiplication of this equation by 2, Eqs. (51) and (53)-(55) must be corrected as follows:

$$
\begin{aligned}
\frac{d}{d t}\left\langle E_{1 k}\right\rangle & =C\left(v_{k}, T_{1 k}\right) \dot{T}_{1 k} \\
& =-\frac{\hbar \hat{\gamma} \Delta D^{2} v_{k}^{2}}{\pi\left(v_{k}^{2}+D^{2}\right)} G\left(v_{k}, t\right)\left[n\left(T_{1 k}, v_{k}\right)-n\left(T_{2 k}, v_{k}\right)\right] \\
C_{k}\left(T_{1 k}\right) \dot{T}_{1 k} & =-\frac{\hat{\gamma} \Delta v_{k} D^{2}}{\pi\left(v_{k}^{2}+D^{2}\right)} G\left(v_{k}, t\right) C_{k}\left(\bar{T}_{k}\right)\left(T_{1 k}-T_{2 k}\right) \\
\dot{T}_{1 k} & =-\frac{\hat{\gamma} \Delta v_{k} D^{2}}{\pi\left(v_{k}^{2}+D^{2}\right)} G\left(v_{k}, t\right)\left(T_{1 k}-T_{2 k}\right)
\end{aligned}
$$

and

$$
\frac{d}{d t} \delta T_{k}=-\frac{2 \hat{\gamma} \Delta v_{k} D^{2}}{\pi\left(v_{k}^{2}+D^{2}\right)} G\left(v_{k}, t\right) \delta T_{k}
$$

The last equation can be reduced to

$$
\frac{d}{d t} \delta T_{k}=-(2 m+1) \hat{\Omega}_{k} \Delta \delta T_{k}
$$

as was explained in the original article where Eq. (76) must be replaced by

$$
\hat{\Omega}_{k}=\frac{2\left(\hat{\gamma} \omega_{k} D^{2}\right)^{2}}{\pi\left(\omega_{k}^{2}+D^{2}\right) \operatorname{den}\left(\omega_{k}\right)} .
$$

In addition, the expression for $e_{i}^{k}$ in Eq. (A3) in the Appendix must be replaced by

$$
\begin{aligned}
e_{i}^{k} & =\frac{\sqrt{2} \Delta \omega_{i}\left|\sin \left[\phi\left(\omega_{k}\right)\right]\right|}{\pi\left(\omega_{i}^{2}-v_{k}^{2}\right)} \sqrt{\frac{D^{2}+v_{k}^{2}}{D^{2}+\omega_{i}^{2}}}, \\
i & =1,2, \ldots, N,
\end{aligned}
$$

where we took into account that $\omega_{1 i}=\omega_{2 i} \equiv \omega_{i}$ due to Eq. (13). Despite the fact that these corrections do not change qualitatively the main results of the article (no revisions of the Abstract and Conclusions are needed), they lead to some modifications of the mode temperatures and thermal current between the reservoirs, which are reflected in Figs. 6-8.

There are also errors in the text right after Eq. (21): there must be $\exp \left(-z_{n} \mathcal{T}\right)$ instead of $\exp \left(-i z_{n} \mathcal{T}\right)$ and $\exp \left(-z_{n}^{\prime} \mathcal{T}\right)$ instead of $\exp \left(-i z_{n}^{\prime} \mathcal{T}\right)$. Finally, factor $\Delta$ must be added to the left hand side of Eq. (67) of the original article. These errors do not propagate anywhere further and do not change any results.

The origin of the factors $1 / 2$ and " $\sqrt{2}$ " in Eqs. (50) and (A3) can be explained in the following way (a more general case is described in Ref. [1]). The interaction matrix in $\mathcal{H}_{\text {tot }}$ can be arranged in such a way that, except its diagonal terms, the only nonzero matrix elements occupy the first line and column. In this case, the matrix is easily diagonalized (analytically), and each eigenfrequency $v_{k}$ is associated with the eigenvector,

$$
e^{k}=e_{0 k}\left[1,\left.E_{i}\left(v_{k}\right)\right|_{i=1} ^{N},\left.E_{i}\left(v_{k}\right)\right|_{i=1} ^{N}\right],
$$

where $\quad E_{i}\left(v_{k}\right)=\omega_{i} \sqrt{C \Delta /\left(\omega_{i}^{2}+D^{2}\right)} /\left(v_{k}^{2}-\omega_{i}^{2}\right) \quad$ and $C=2 \hat{\gamma} D^{2} / \pi$. The first component in the square brackets in Eq. (1) corresponds to the molecule, the first set of $E_{i}\left(v_{k}\right)$ corresponds to the left reservoir, and the second one corresponds to the right reservoir. Normalization factor $e_{0 k}$ can be found as

$$
\begin{aligned}
\left(e_{0 k}\right)^{-2} & =1+2 C \sum_{i=1}^{N} \frac{\Delta \omega_{i}^{2}}{\left(\omega_{i}^{2}+D^{2}\right)\left(v_{k}^{2}-\omega_{i}^{2}\right)^{2}} \\
& \approx 1+\frac{2 C v_{k}^{2}}{v_{k}^{2}+D^{2}} \sum_{i=1}^{N} \frac{\Delta}{\left(v_{k}^{2}-\omega_{i}^{2}\right)^{2}} \\
& \approx \frac{\pi^{2} C}{2 \Delta\left(v_{k}^{2}+D^{2}\right) \sin ^{2}\left(\phi_{k}\right)}
\end{aligned}
$$

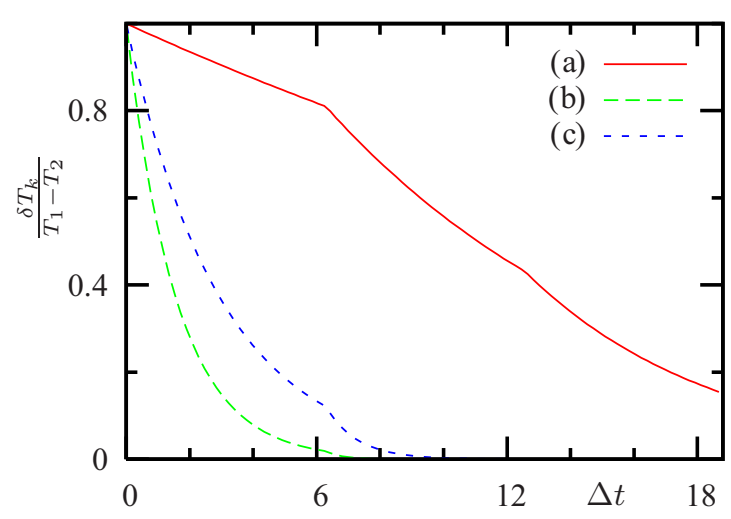

FIG. 6. Temperature relaxation curves produced by solving Eq. (75) at $\hat{\gamma} / \omega_{0}=0.5, D / \omega_{0}=1$, and $\Delta / \omega_{0}=0.001$. (a) $v_{k}=$ $0.5 \omega_{0}$, (b) $v_{k}=v_{\text {res }} \approx \omega_{0}$, and (c) $v_{k}=1.1 \omega_{0}$. 


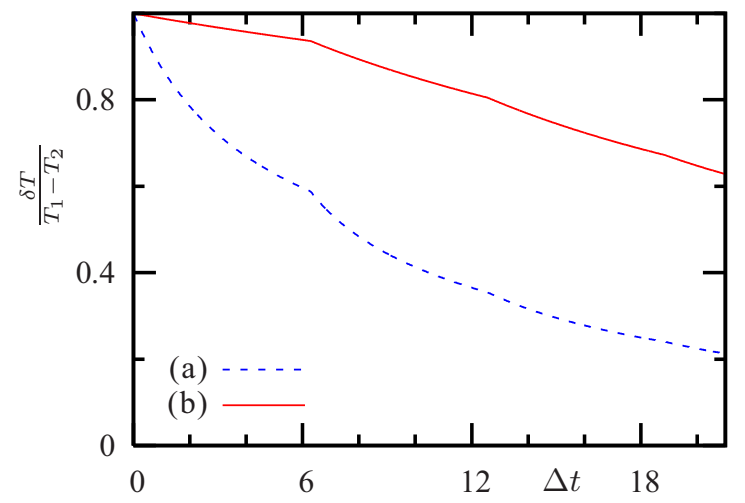

FIG. 7. Average temperature relaxation curves when $\hat{\gamma} / \omega_{0}=$ $0.5, D / \omega_{0}=1$, and $\Delta / \omega_{0}=0.001$. (a) $\hbar \omega_{0} / 2 k_{\mathrm{B}} \bar{T}=0.1$ and (b) $\hbar \omega_{0} / 2 k_{\mathrm{B}} \bar{T}=5$.

where $\phi_{k}$ is determined by (A2) and (25) in the article. Here, we used the relation [2],

$$
\sum_{i=1}^{N} \frac{\Delta}{\left(v_{k}^{2}-\omega_{i}^{2}\right)^{2}} \approx \frac{\pi^{2}}{4 \Delta v_{k}^{2} \sin ^{2}\left(\phi_{k}\right)}
$$

and neglected " 1 " compared to $\Delta^{-1}$. As our numerics show, the relative error of relation (2) is $\lesssim 10^{-3}$ if $\Delta \lesssim 10^{-3}$. After substituting $e_{0 k}$ into the above expression for $e^{k}$, one arrives at Eq. (A3).

Substituting (A1) and $p_{v i}(t)=m_{i} \dot{x}_{v i}(t)$ into expression

$$
E_{v}=\sum_{i=1}^{N}\left\langle\frac{p_{v i}^{2}}{2 m_{i}}+\frac{m_{i} \omega_{i}^{2} x_{v i}^{2}}{2}\right\rangle
$$

for the averaged energy of the $v$ th reservoir, dropping contributions proportional to $a_{v k}^{+} a_{v k_{1}}^{+} \exp \left[i\left(v_{k}+v_{k_{1}}\right) t\right]$ and $a_{v k} a_{v k_{1}} \exp \left[-i\left(v_{k}+v_{k_{1}}\right) t\right]$ yielding zero at time averaging due

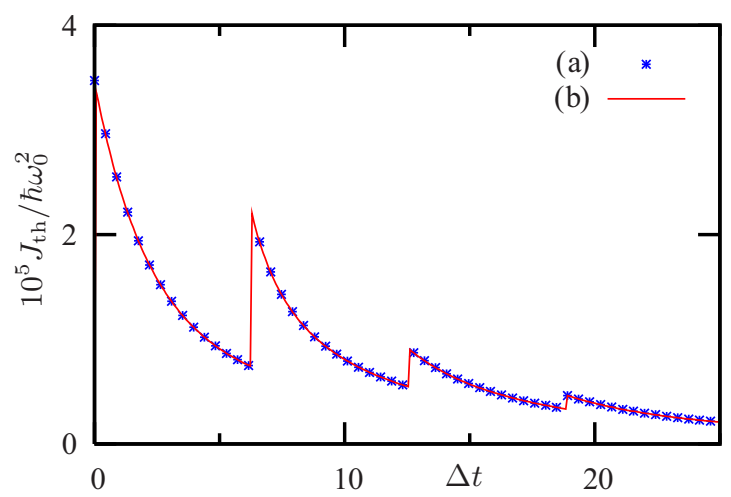

FIG. 8. Dimensionless thermal current curves when $\hat{\gamma} / \omega_{0}=$ $0.5, D / \omega_{0}=1, \Delta / \omega_{0}=0.001, \hbar \omega_{0} / 2 k_{\mathrm{B}} \bar{T}=1$, and (a) approximation (69) is used; (b) the accurate expression (65) is used.

to their fast time dependences, and taking into account the eigenmode independence, one finds

$$
E_{v}=\frac{\hbar}{4}\left[\sum_{k} v_{k} n_{\nu k} \sum_{i=1}^{N}\left(e_{i}^{k}\right)^{2}+\sum_{k} \frac{n_{\nu k}}{v_{k}} \sum_{i=1}^{N}\left(\omega_{i} e_{i}^{k}\right)^{2}\right] .
$$

Using (A3) (corrected here), we find in a similar way as in deriving expression (2) and with the same accuracy,

$$
\sum_{i=1}^{N}\left(e_{i}^{k}\right)^{2} \approx v_{k}^{-2} \sum_{i=1}^{N}\left(\omega_{i} e_{i}^{k}\right)^{2} \approx \frac{1}{2}
$$

and, taking into account relation $\left\langle E_{v k}\right\rangle=\hbar v_{k} n_{v k} / 2=$ $\hbar v_{k}\left\langle a_{k}^{+} a_{k}+a_{k} a_{k}^{+}\right\rangle / 2$, one finds

$$
E_{v}=\frac{\hbar}{4} \sum_{k} v_{k} n_{v k}=\frac{1}{2} \sum_{k}\left\langle E_{v k}\right\rangle, \quad v=1 \text { or } 2,
$$

which gives us the correct Eqs. (50), (51), (53)-(55), and (76).
[1] G. Y. Panasyuk, K. L. Yerkes, and T. J. Haugan, Phys. Rev. E 99, 032141 (2019).
[2] A. P. Prudnikov, Y. A. Brychkov, and O. I. Marichev, Integrals and Series: Elementary Functions (Gordon and Breach, Amsterdam, 1998), Vol. 1. 